\title{
ARTÍCULOS
}

\section{FACT CHECKING: UN NUEVO DESAFÍO DEL PERIODISMO}

\section{Fact checking: A new challenge in journalism}

\section{María-José Ufarte-Ruiz, Lidia Peralta-García y Francisco-José Murcia-Verdú}

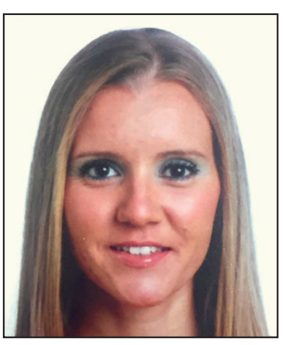

María-José Ufarte-Ruiz es doctora en Periodismo por la Universidad de Sevilla y profesora en la Universidad de Castilla-La Mancha. Es autora de los libros Twitter como herramienta para la comunicación política electoral (2017); Preguntar para escribir (2016); y Un oficio que se nos va (2015). Ha participado en proyectos de I+D+i y cuenta con varias estancias académicas. Pertenece al grupo de investigación Influencias de los Géneros Periodísticos y de las Tecnologías en la Comunicación Social.

https://orcid.org/0000-0002-7713-8003

Universidad de Castilla-La Mancha, Campus Cuenca. Polivalente, Campus Universitario, s/n. 16071 Cuenca, España mariajose.ufarte@uclm.es

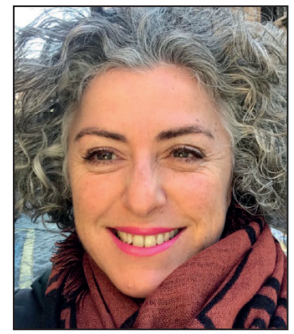

Lidia Peralta-García es profesora ayudante doctora en la Facultad de Periodismo de Cuenca (Universidad de Castilla-la Mancha). Doctora en Periodismo por la Universidad de Málaga, es miembro del grupo de investigación Gabinete de Comunicación y Educación de la Universidad Autónoma de Barcelona (UAB), donde impartió clases desde 2013 a 2016. Imparte clases en el Master de Comunicación y Educación y en el Master de Periodismo de Viajes de la UAB. Es autora del libro Los nuevos héroes del siglo XXI. Las migraciones subsaharianas vistas por el cine en España y África. https://orcid.org/0000-0003-2934-0108

Universidad de Castilla-La Mancha, Campus Cuenca. Polivalente, Campus Universitario, s/n. 16071 Cuenca, España lidia.peralta@uclm.es

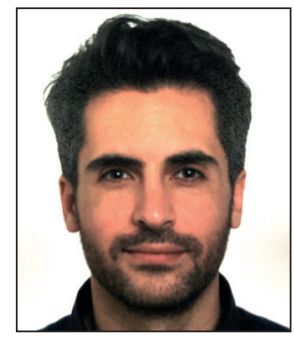

Francisco-José Murcia-Verdú es licenciado en Periodismo por la Universidad del País Vasco, doctorando por la misma universidad, y alumno del Master de Innovación en Periodismo de la Universidad Miguel Hernández. Es redactor en el Diario de Almería (Grupo Joly), autor de numerosos capítulos de libro de divulgación periodística y cuenta con cursos de especialización. Su campo de investigación es la influencia de las tecnologías en los géneros periodísticos, y la relación entre periodismo y literatura.

https://orcid.org/0000-0001-6020-1689

Universidad del País Vasco Facultad de Ciencias Sociales y de la Comunicación Bo Sarriena, s/n. 48940 Leioa (Bizkaia), España franmurciaverdu@gmail.com

\section{Resumen}

Se analizan las similitudes y discrepancias entre el discurso académico y el profesional en torno al fact checking, entendiéndolo desde una doble perspectiva, tanto como una competencia que el actual periodista tiene que adquirir, como un campo de trabajo que ofrece nuevas oportunidades. Se hace una revisión bibliográfica sobre la figura del verificador de datos, se recaba la opinión de siete profesores universitarios mediante una entrevista estructurada, y conoce la postura de los estudiantes a través de un cuestionario de preguntas cerradas y categóricas $(n=316)$. También se analiza el contenido de las unidades de verificación de datos que hay en España con el fin de conocer cuáles son las competencias más demandadas. Los resultados reflejan que el debate sigue abierto, al mismo tiempo que muestran una coincidencia en ambos discursos acerca de la necesidad de una sólida formación de los periodistas que integren nuevos perfiles.

\section{Palabras clave}

Fact checking; Verificación; Noticias falsas; Credibilidad; Perfiles periodísticos; Cibermedios; Tecnologías de la información y la comunicación; TIC; Periodismo. 


\begin{abstract}
The present article analyses similarity and differences between academic and professional speech about "fact checking", understanding it from a double perspective, both, like a competence that current journalists have to acquire, and a work field that offers new opportunities. The research is based on a bibliographical review about the fact checkers' profiles. It also collects the opinion of seven university professors by means of a structured interview and has access to the student's point of view through a close-response test and a categorical test $(n=316)$. It also analyses the content of the data verification departments that exists in Spain with the aim of knowing which the most demanded competences are. The results reflect that the discussion is still open and at the same time it shows a coincidence between both speeches about the necessity of a journalist' solid training that integrates new profiles.
\end{abstract}

\title{
Keywords
}

Fact checking; Verification; Fake news; Credibility; Journalistic profiles; Cybermedia; Online newspapers; Information and Communication Technologies; ICT; Journalism.

Ufarte-Ruiz, María-José; Peralta-García, Lidia; Murcia-Verdú, Francisco-José (2018). "Fact checking: un nuevo desafío del periodismo". El profesional de la información, v. 27, n. 4, pp. 733-741.

https://doi.org/10.3145/epi.2018.jul.02

\section{Introducción}

La crisis de la industria periodística y la precariedad han llevado al profesional de la información a practicar un periodismo de mesa alejado de la calle. Entre 2008 y 2015 los medios de comunicación han realizado numerosas reestructuraciones y han destruido más de 12.200 empleos (APM, 2017, p. 24). La concatenación de la crisis publicitaria, empresarial y profesional ha devaluado la actividad periodística. Las iniciativas y proyectos emprendedores en formato digital se han multiplicado (Manfredi-Sánchez; Rojas-Torrijos; Herranz-de-la-Casa, 2015; Casero-Ripollés; Cullell-March, 2013), y los contenidos de baja calidad pero alto impacto en la audiencia se han disparado. La viralización de titulares sensacionalistas ha banalizado el periodismo y afectado a su credibilidad. Como apunta Ignacio Escolar:

"la prensa sufre una crisis de credibilidad como la de las instituciones" (eldiario.es, 2016).

En este contexto, la difusión de informaciones falsas e imprecisas que inducen a error a los lectores está en auge y se ha convertido en una amenaza cada vez mayor para las democracias europeas. La Comisión Europea (2018) ha creado incluso un grupo de alto nivel compuesto por 39 expertos para afrontar el problema de las noticias falsas y desinformación en línea.

Las nuevas tecnologías han cambiado la forma de consumir la información y hace tiempo que se habla sobre la muerte del papel. Los medios digitales llevan años funcionando y el imperio de las redes sociales se ha sobrepuesto a los medios convencionales. Así lo entiende Massot (2017), que considera que las informaciones circulan tan masiva y aceleradamente en las redes sociales que tendemos a agarrarlas y retransmitirlas con el mismo instinto compulsivo que el de un jugador de ping-pong: las capta y rebota, sin detenerse a comprobar su veracidad. En términos similares se pronuncian Ciampaglia et al. (2015), que sostienen que la comprobación de hechos tradicional por parte de periodistas expertos no puede mantenerse al día con el enorme volumen de información que ahora se genera online.
La necesidad de atajar este problema ha dado lugar al fact checking, también conocido como verificación de datos, una operación que aplica técnicas del periodismo de datos para desenmascarar los errores, ambigüedades, mentiras, falta de rigor o inexactitudes de algunos contenidos publicados en los medios de comunicación. Se trata de un filtro que garantiza que los textos periodísticos están contrastados a partir de fuentes fiables, documentos oficiales y resultados de investigaciones solventes, en un momento en el que la profesión periodística está lastrada por la precariedad laboral (Fole, 2012; Gómez-Mompart; Gutiérrez-Lozano; Palau-Sampio, 2015; Álvarez-Gromaz; López-García, 2016; López-García; Rodríguez-Vázquez; Álvarez-Gromaz, 2016; Redondo, 2017; Amazeen, 2017). Son las herramientas tecnológicas las que, mediante procesos de automatización, contribuyen a optimizar estos resultados y garantizar la excelencia (López-García; Toural-Bran; Rodríguez-Vázquez, 2016). Ana Pastor explica:

"es una apuesta necesaria para que la gente cuando comparta una noticia pueda saber si es cierta o falsa ( $L a$ sexta, 2017).

\section{El fact checking garantiza que los textos periodísticos estén contrastados a partir de fuentes fiables, documentos oficiales y resultados de investigaciones solventes}

Desde la primera década del siglo XXI las propuestas de fact checking se han expandido tanto a nivel nacional como internacional, a pesar de que la labor de confirmar y comprobar hechos existe desde el inicio del periodismo. También se han diferenciado diversos tipos de verificación (Miniver, Ministerio de la verdad) y se aconseja sobre cómo ejercer estas funciones (Red Internacional de Verificación de Información, IFCN). En la práctica, varios medios españoles han fortalecido y creado sus propias unidades de verificación, una iniciativa que ha servido de estímulo para que se creen otros proyectos periodísticos independientes dedicados ex- 
clusivamente a esta actividad, sobre todo en internet. La tabla 1 recoge propuestas que existen en el país.

La bibliografía sobre fact checking es escasa (Wintersieck, 2017) debido a su novedad y poco volumen, y los métodos utilizados para la comprobación de datos son insuficientes (Uscinski; Butler, 2013). Por eso habría que dotar a los futuros periodistas de unas competencias trasversales para la solución de problemas específicos (Manfredi-Sánchez, 2015), ya que la llegada de los datos masivos (big data) ha abierto nuevas perspectivas para los profesionales de la información (Meyer, 1991; Elías-Pérez, 2015; Crucianelli, 2012).

La presente investigación plantea el siguiente objetivo: analizar las similitudes y discrepancias entre el discurso académico y el profesional en torno al fact checking. El trabajo recurre tanto a la metodología cuantitativa como cualitativa y parte de las siguientes hipótesis:

H1. En el ámbito académico los docentes en Periodismo consideran que el fact checking es una obligación en el ejercicio de la clase periodística y un requisito inexcusable para la elaboración de piezas de calidad, como también apuntan Kovach y Rosenstiel (2001) y la Fundación del Español Urgente, Fundéu BBVA, (2013).

H2. En el ámbito profesional la realidad ha empujado a los medios a la búsqueda de iniciativas destinadas a contrastar las informaciones. Este interés también existe entre los estudiantes, que apuestan por verificar datos como una nueva oportunidad laboral.

En las siguientes líneas se recoge la bibliografía científica para entender el contexto, se presenta la metodología y se explican los resultados obtenidos en la presente investigación.

\section{Antecedentes: las noticias falsas}

Con la eclosión de las redes sociales se ha introducido un elemento de distorsión, de peligro a futuro, si no se ataja con la máxima rapidez. Son las llamadas noticias falsas (fake news, según la terminología inglesa), que define la información distorsionada (Khaldarova; Pantti, 2016), con altos niveles de facticidad y engaño (Tandoc; Lim; Ling, 2017), que se modifica, se ridiculiza y se somete a nuevas interpretaciones (Balmas, 2012). Se trata de una vieja práctica que ya se usaba en algunos panfletos y publicaciones del siglo XVIII y $\mathrm{XIX}$, pero que cambia con internet al acelerarse la distribución de la información y socializarse el contenido masivamente (Nelson; Harsh, 2018; Haigh; Haigh; Kozak, 2017). Según explica Rosell, director de El mundo:

"Las fake news revalorizan, revitalizan el papel del periodismo ya que las redes sociales se han comido a las noticias, amenazan la viabilidad del periodismo basado en el interés público y han contribuido a una era en la que las opiniones están sustituyendo a los hechos" (Europa Press, 2017).

Las noticias falsas han existido desde tiempo inmemorial (Wilkinson, 2017), aunque empezaron a sonar con más frecuencia después de que Trump fuera elegido presidente de EUA. El republicano utilizó el término durante su campaña electoral para poner en duda la veracidad de las informa-
Tabla 1. Unidades activas de verificación de datos en España

\begin{tabular}{|c|c|c|}
\hline Año & Nombre & Tipo de proyecto \\
\hline 2004 & $\begin{array}{l}\text { Malaprensa } \\
\text { Josu Mezo }\end{array}$ & Independiente \\
\hline 2006 & $\begin{array}{l}\text { Miniver (Ministerio de la verdad) } \\
\text { Alfredo Expósito }\end{array}$ & Independiente \\
\hline 2013 & $\begin{array}{l}\text { Maldita hemeroteca } \\
\text { Clara Jiménez y Julio Montes }\end{array}$ & Independiente \\
\hline 2015 & $\begin{array}{l}\text { Maldito bulo } \\
\text { Clara Jiménez y Julio Montes }\end{array}$ & Independiente \\
\hline 2015 & $\begin{array}{l}\text { La chistera } \\
\text { El confidencial }\end{array}$ & Depende del medio \\
\hline 2016 & $\begin{array}{l}\text { Verdad o mentira } \\
\text { Periodista digital }\end{array}$ & Depende del medio \\
\hline 2017 & $\begin{array}{l}\text { El objetivo } \\
\text { La sexta }\end{array}$ & Depende del medio \\
\hline 2017 & $\begin{array}{l}\text { El cazabulos } \\
\text { eldiario.es }\end{array}$ & Depende del medio \\
\hline 2017 & $\begin{array}{l}\text { Hechos } \\
\text { El país }\end{array}$ & Depende del medio \\
\hline 2017 & $\begin{array}{l}\text { El tragabulos } \\
\text { Verne, El país }\end{array}$ & Depende del medio \\
\hline
\end{tabular}

ciones de algunos medios (Jankowski, 2018; Kleis-Nielsen; Graves, 2016; Greenberg, 2017). También han jugado un papel destacado en el referéndum del Brexit, o en las presidenciales francesas, y han vuelto a cobrar protagonismo en la crisis catalana, con Rusia como protagonista. A los dirigentes europeos les preocupa que estas interferencias se repitan en las elecciones a la Eurocámara de mayo de 2019 (Sanhermelando, 2018).

Lo que surgió como un concepto político, ha acabado extrapolándose a otros campos, minando la credibilidad del conjunto de las noticias que circulan. De hecho, en España existen varias webs que propagan noticias falsas a través de redes sociales y consiguen cientos de miles de visitas. Es el caso, por ejemplo, de Mediterráneo digital, Veterinarios. info o Cerebrother, que ha llegado a facturar en un mes casi 4.000 euros (Llaneras; Pérez-Colomé, 2017).

Aunque diversos estudios señalan que el poder de las fake news es "poco desestabilizador" (Hazard-Owen, 2018; Carey, 2018; Margolin; Hannak; Weber, 2017), la práctica profesional evidencia la necesaria labor de combatirlas. Para ello se han lanzado sistemas de fact checking o verificación de datos, que desarrollan y fortalecen prácticas para garantizar una cobertura informativa correcta (Greenberg, 2017).

\section{Metodología}

El trabajo utiliza un método multidisciplinar que combina técnicas de investigación cualitativas y cuantitativas, como las entrevistas estructuradas, el análisis de contenido y el cuestionario.

En primer lugar, se han realizado entrevistas estructuradas a siete profesores universitarios. Dos son expertos en noticias falsas y verificación de datos:

- Miguel-Ángel Benedicto-Solsona: Universidad de Comillas-Icade, de Madrid; 
- Juan-Luis Manfredi-Sánchez: Universidad de Castilla-La Mancha.

Los otros cinco entrevistados son:

- Carmen Peñafiel-Saiz: de la Universidad del País Vasco;

- Antonio López-Hidalgo: Universidad de Sevilla;

- Margarita Cabrera-Méndez: Universidad Politécnica de Valencia;

- Miguel Carvajal-Prieto: Universidad Miguel Hernández de Elche;

- Félix Robles-Arias: Universidad Miguel Hernández de Elche.

La elección de esta muestra responde al impacto que ha tenido la obra de los profesores seleccionados en el campo del periodismo, la innovación, la tecnología y en los asuntos de orden político.

En segundo lugar, hemos analizado todas las plataformas de verificación de datos que hay en España (tabla 1). Este estudio está dirigido a esclarecer qué requisitos formativos y actitudinales son los más requeridos para ejercer la verificación de datos.

Por otro lado, hemos realizado un cuestionario con diez preguntas cerradas y categóricas relacionadas con el objeto de estudio. La recogida de datos se llevó a cabo entre los meses de enero y marzo de 2018. Los resultados de esta investigación se han obtenido a partir de una muestra mixta de entrevistados (tabla 2).

Los medios son conscientes de la importancia de la verificación de datos para alcanzar un periodismo de calidad y, como consecuencia, los proyectos periodísticos destinados a esta actividad se han disparado en los últimos años

Siguiendo el modelo planteado por Humanes-Humanes y Roses-Campos (2014), hemos usado un criterio de conveniencia basado en nuestra red de colaboración académica para la elección de las tres universidades públicas donde se ha realizado la encuesta. Se ha seleccionado a estudiantes de diferentes cursos para comprobar si las percepciones sobre el fact checking varían en función de los conocimientos adquiridos a lo largo del grado (Casero-Ripollés; Ortells-Badenes; Doménech-Fabregat, 2013). Debido a las características de la muestra, los resultados no son completamente generalizables al no estar representadas, por ejemplo, las universidades privadas y otros territorios del Estado. No por ello, quedan invalidados, puesto que la finalidad primordial de este trabajo no es de carácter cuantitativo sino cualitativo. Es decir, no importa tanto el número como los argumentos que emplean los futuros periodistas e implicados más directamente en el objeto de estudio.

\section{Resultados}

\subsection{Perspectivas sobre la verificación de datos como salida laboral}

El fact checking goza de gran aceptación entre los estudiantes de Periodismo. El 70,69\% de los alumnos sabe qué es y para qué sirve, frente al $29,31 \%$ que desconoce su significado más allá de su traducción. De este porcentaje, el 19,40\% cursa el primer año del grado en Periodismo, mientras que el resto de estudiantes, pertenece al segundo $(5,43 \%)$ y tercer curso $(4,48 \%)$.

Respecto a si es una nueva oportunidad laboral, un $94,54 \%$ confirma que sí y un 71,42\% trabajaría exclusivamente verificando datos. A tenor de estas cifras, Margarita Cabrera-Méndez (2018) recuerda que hay proyectos que están funcionando muy bien, como Chequeado en Argentina. Por el contrario, un $5,46 \%$ defiende que las tareas que ejercen forman parte del trabajo de cualquier medio de comunicación. Esta postura es compartida por Miguel-Ángel Benedicto-Solsona (2018), que apunta: "Verificar datos y fuentes fiables debe formar parte del abecé del periodista". Carmen Peñafiel-Saiz (2018) también recuerda que la verificación de hechos y datos ha sido una obligación en el ejercicio de la clase periodística durante todos los tiempos y Antonio López-Hidalgo (2018) sostiene que, si lo consideramos una salida profesional, "significaría que la profesión se ha pervertido".

Sobre si los verificadores de datos juegan un papel importante a la hora de recuperar la credibilidad de los medios, un $87,56 \%$ opina que son un requisito esencial, frente al $12,44 \%$ restante que cree que la imagen del periodismo español ya está dañada y poco puede hacerse para restaurarla. Una posible fórmula apunta hacia las fuentes y la metodología del trabajo. El 96,55\% de los estudiantes afirma que las fuentes periodísticas son fundamentales en la credibilidad y transparencia, mientras que otros explican que nunca son imparciales (3,45\%). Sin embargo, para Félix Arias-Robles (2018) "no hay una fórmula mágica", ya que a esta "receta" hay que sumar

"múltiples herramientas que permiten, por ejemplo, verificar la autenticidad de una foto o calcular el ámbito de influencia de una fuente". 
A los alumnos también se les preguntó si proporcionarían a los lectores todos los datos relativos a las fuentes informativas para que puedan replicar el trabajo periodístico y aproximadamente la mitad $(48,28 \%)$ contestaron que sí, siempre que no les comprometa.

Un $94,5 \%$ de los alumnos $(n=316)$ confirma que el fact checking es una nueva oportunidad laboral y un $71,4 \%$ trabajaría exclusivamente verificando datos

En cuanto a ser transparentes con la metodología utilizada para seleccionar e investigar un acontecimiento, un 56,90\% cree que hay que tener en cuenta la inmediatez y periodicidad del medio. Como consecuencia, Miguel Carvajal-Prieto (2018) recuerda que

"siempre hay que revisar el método de obtención de la información, contrastar la procedencia del material, examinar los intereses de las fuentes, comprobar y revisar dos veces el contenido y no confiar en el material ajeno obtenido de medios o fuentes desconocidas en redes sociales".

Sin embargo, el panorama cambia cuando se les pregunta si corregirían una información de acuerdo con la política de rectificaciones del medio de comunicación. El 82,76\% asevera que sí, pues los lectores deben saber la verdad, mientras que el $17,24 \%$ contesta que no. De este porcentaje, un $15,20 \%$ considera que su credibilidad está en juego, mientras que el otro $2,04 \%$ no desvela ninguna razón.

Tabla 3. Perfiles según el tipo de empresa

\begin{tabular}{|c|c|}
\hline Malaprensa & $\begin{array}{l}\text { Ciencias Políticas } \\
\text { Derecho } \\
\text { Ciencias Sociales }\end{array}$ \\
\hline Miniver & $\begin{array}{l}\text { Derecho } \\
\text { Periodistas }\end{array}$ \\
\hline Maldita hemeroteca & $\begin{array}{l}\text { Periodistas de datos } \\
\text { Ingenieros informáticos } \\
\text { Desarrolladores de plataformas web y aplicaciones móviles }\end{array}$ \\
\hline Maldito bulo & $\begin{array}{l}\text { Periodistas de datos } \\
\text { Ingenieros informáticos } \\
\text { Desarrolladores de plataformas web y aplicaciones móviles }\end{array}$ \\
\hline $\begin{array}{l}\text { La chistera } \\
\text { El confidencial }\end{array}$ & Periodistas de datos \\
\hline $\begin{array}{l}\text { Verdad o mentira } \\
\text { Periodista digital }\end{array}$ & Periodistas \\
\hline $\begin{array}{l}\text { El objetivo } \\
\text { La sexta }\end{array}$ & $\begin{array}{l}\text { Periodistas } \\
\text { Periodistas de datos } \\
\text { (cuenta con la colaboración de los perfiles de Maldito bulo) }\end{array}$ \\
\hline $\begin{array}{l}\text { El cazabulos } \\
\text { eldiario.es }\end{array}$ & $\begin{array}{l}\text { Periodistas } \\
\text { Investigadores sociales } \\
\text { (cuenta con la colaboración de los perfiles de Maldito bulo) }\end{array}$ \\
\hline $\begin{array}{l}\text { Hechos } \\
\text { El país }\end{array}$ & $\begin{array}{l}\text { Periodistas } \\
\text { Periodistas de datos }\end{array}$ \\
\hline $\begin{array}{l}\text { El tragabulos } \\
\text { Verne, El país }\end{array}$ & $\begin{array}{l}\text { Periodistas } \\
\text { Periodistas de datos }\end{array}$ \\
\hline
\end{tabular}

\subsection{Perfiles y competencias}

Existe una hibridación de perfiles y una transversalidad de conocimientos, aptitudes y actitudes en torno al fact checking que revela la necesidad de formar en nuevas competencias adaptadas al impacto de la innovación tecnológica, de acuerdo a lo que ya proponían López-García, Rodríguez-Vázquez y Pereira-Fariña (2017); Besalú-Casademont, Schena y Sánchez-Sánchez (2017) y Casero-Ripollés, Ortells-Badenes y Doménech-Fabregat (2013). Las competencias se refieren a conocimientos para proporcionar servicios concretos, al manejo de aplicaciones y a formas y actitudes para afrontar la profesión (Álvarez-Flores; Núñez-Gómez; Olivares-Santamarina, 2018). En la tabla 3 se especifican los perfiles según el tipo de empresa.

\section{Hay que dotar a los futuros periodistas de unas competencias trasversales, ya que la llegada de los datos masivos ha abierto nuevas perspectivas}

El análisis pormenorizado demuestra que la mayoría de los perfiles clásicos conviven con nuevas funciones periodísticas. La mayoría de las iniciativas de fact checking apuestan por una especialización en big data, es decir, en datos masivos que estén presentes en internet y que sirven como fuente de análisis, interpretación y reconstrucción de la información (Renó; Flores, 2014). También deben dominar las redes sociales, de acuerdo con González-Molina y Ortells-Badenes (2012). El manejo de estas destrezas puede mejorar la empleabilidad de los futuros graduados en Periodismo, ya que muchos jóvenes tienen dificultades para encontrar trabajo debido al desajuste entre su capacitación y los requerimientos del mercado (Bremer, 2013). Otros autores (Álvarez-Flores; Núñez-Gómez; Rodríguez-Crespo, 2017; Sánchez-González; Méndez-Muros, 2013; Alonso-Benito; Fernández-Rodríguez; Nyssen-González, 2009) también confirman que las competencias de los estudiantes o graduados son distintas a las que piden las empresas.

Juan-Luis Manfredi-Sánchez resalta la necesidad de ajustar la formación de los futuros periodistas a las funciones requeridas por el sector, de acuerdo con Félix Arias-Robles y Farias-Batlle (2009). Carmen Peñafiel-Saiz indica:

“Debemos formar mejor a los periodistas y a quienes se dediquen a verificar informaciones o datos, para que analicen correctamente casos de falsedad, errores, mentiras".

Su postura es compartida por Miguel-Ángel Benedicto-Solsona, que también apuesta por renovar los planes de estudios para conciliar formación y realidad profesional, 
como también señalan García-Valcárcel-Muñoz-Repiso y Martín-del-Pozo (2016); Torres-Coronas y Vidal-Blasco (2015); Armendáriz (2015); Perlado-Lamo-de-Espinosa y Rubio-Romero (2015); Arias-Oliva, Torres-Coronas y Yález-Luna (2014); Martín-del-Peso, Rabadán-Gómez y Hernández-March (2013).

Miguel Carvajal-Prieto aboga por una especialización en los métodos y en la selección de las fuentes, mientras que Margarita Cabrera-Méndez apuesta por fomentar el manejo de herramientas de fact checking.

Por el contario, Antonio López-Hidalgo considera que la única función del periodista "es contrastar y escribir con calidad de estilo". Al igual que sugieren Besalú-Casademont, Schena y Sánchez-Sánchez (2017), existen discrepancias entre académicos y profesionales a la hora de valorar qué competencias son más importantes, reforzándose asimismo conclusiones similares a las de otros estudios (García-Ureta; Toral-Madariaga; Murelaga-Ibarra, 2012).

Las aportaciones subrayan, además, la necesidad de establecer una "doble vía" que refuerce el conocimiento de los elementos básicos del periodismo y los combine con la capacitación tecnológica (López-García; Rodríguez-Vázquez; Pereira-Fariña, 2017).

La mayoría de los perfiles clásicos conviven con nuevas funciones periodísticas

\section{Discusión y conclusiones}

La investigación realizada permite dar por conseguido el objetivo planteado inicialmente de contrastar la postura de académicos y profesionales sobre el fact checking.

Permite confirmar la primera hipótesis, ya que los profesores universitarios participantes consideran que la verificación de datos ha de ser una competencia propia de los periodistas, un deber en el ejercicio de la profesión.

También es posible confirmar la segunda hipótesis, debido a que los medios son cada vez más conscientes de su importancia para alcanzar un periodismo de calidad y, como consecuencia, los proyectos periodísticos destinados a esta actividad se han disparado en los últimos años. Los estudiantes de Periodismo también empiezan a ver a los verificadores de datos como una oportunidad laboral y asumen sus tareas con naturalidad. Así lo revela la encuesta realizada a un total de 316 alumnos, de los que un $94,54 \%$ considera que es una nueva salida profesional y un $71,42 \%$ trabajaría exclusivamente verificando datos.

A tenor de estas cifras, los docentes apuestan por revisar las necesidades formativas para adaptarlas a los nuevos perfiles en la era digital (López-García, 2012; Palomo, 2013), ya que los medios demandan nuevas competencias y habilidades derivadas de la tecnologización. Hay que afrontar la necesidad de preparar a los periodistas para un entorno cambiante en el que las tecnologías marcan las transformaciones, lo que obliga a entender su planteamiento y sus singularidades, pero sin descuidar los fundamentos periodísticos, aquellos se han sedimentado a lo largo del tiempo
(López-García; Rodríguez-Vázquez; Pereira-Fariña, 2017).

La cuestión estudiada no se agota en esta investigación sino que, muy al contrario, reafirma que se trata de un debate abierto y en evolución. En este proceso se hace patente la conveniencia de explorar nuevas vías de encuentro para redactar una 'hoja de ruta' que permita buscar más puntos de convergencia entre el ámbito profesional y el científicoacadémico en pos del mejor Periodismo posible en una realidad comunicativa cada vez más compleja. En esta 'hoja de ruta' podríamos indicar, por ejemplo, qué proyectos son sostenibles, la innovación en nuevas narrativas, los modelos de negocio existentes y los proyectos independientes y asociados, entre otros puntos.

Hay que fomentar una formación que refuerce el conocimiento de los elementos básicos del periodismo y los combine con la capacitación tecnológica

\section{Entrevistas}

Arias-Robles, Félix: 25 de enero de 2018.

Benedicto-Solsona, Miguel-Ángel: 5 de febrero de 2018.

Cabrera-Méndez, Margarita: 30 de enero de 2018.

Carvajal-Prieto, Miguel: 25 de enero de 2018.

López-Hidalgo, Antonio: 12 de febrero de 2018.

Manfredi-Sánchez, Juan-Luis: 10 de febrero de 2018.

Peñafiel-Sáiz, Carmen: 17 de febrero de 2018.

\section{Referencias}

Alonso-Benito, Luis-Enrique; Fernández-Rodríguez, Carlos-Jesús; Nyssen-González, José-María (2009). El debate sobre las competencias. Una investigación cualitativa en torno a la educación superior y el mercado de trabajo en España. Madrid: Aneca. ISBN: 9788469173947 https://dialnet.unirioja.es/servlet/libro?codigo $=405703$

Álvarez-Flores, Erika-Patricia; Núñez-Gómez, Patricia; Olivares-Santamarina, José P. (2018). "Perfiles profesionales y salidas laborales para graduados en Publicidad y Relaciones Públicas: De la especialización a la hibridación". El profesional de la información, v. 27, n. 1, pp. 136-147.

https://doi.org/10.3145/epi.2018.ene.13

Álvarez-Flores, Erika-Patricia; Núñez-Gómez, Patricia; Rodríguez-Crespo, Carlos (2017). “Adquisición y carencia académica de competencias tecnológicas ante una economía digital". Revista latina de comunicación social, v. 72, pp. 540-559. https://doi.org/10.4185/RLCS-2017-1178

Álvarez-Gromaz, Lucía; López-García, Xosé (2016). “El fact checking como herramienta de combate contra el sensacionalismo". En: Esteban, Lucía (coord.); Almuiña-Fernández, Celso-Jesús (dir.); Martín-de-la-Guardia, Ricardo (dir.); Vidal-Pelaz-López, José (dir.). Sensacionalismo y amarillismo en la historia de la comunicación, pp. 215-228. Madrid: Editorial Fragua. ISBN: 9788470747298 
Amazeen, Michelle A. (2017). "Journalistic interventions: The structural factors affecting the global emergence of fact-checking". Journalism, Sept. $8^{\text {th }}$.

https://doi.org/10.1177/1464884917730217

Arias-Oliva, Mario; Torres-Coronas, Teresa; Yález-Luna, Juan-Carlos (2014). "El desarrollo de competencias digitales en la educación superior". Historia y comunicación social, v. 19, pp. 355-366.

https://doi.org/10.5209/rev_HICS.2014.v19.44963

Armendáriz, Enrique (2015). "El nuevo perfil del profesional de la comunicación y las relaciones públicas. Una visión desde la perspectiva del mercado". Revista internacional de relaciones públicas, v. 5, n. 9, pp. 153-178.

https://doi.org/10.5783/RIRP-9-2015-09-153-178

Balmas, Meital (2012). "When fake news becomes real. Combined exposure to multiple news sources and political attitudes of inefficacy, alienation, and cynicism". Communication research, v. 41, n. 3, pp. 430-454 https://doi.org/10.1177/0093650212453600

Besalú-Casademont, Reinald; Schena, Jucinara; SánchezSánchez, Cristina (2017). “Competencias más relevantes en los estudios de Comunicación Audiovisual. Las percepciones de profesionales, docentes y estudiantes". Revista latina de comunicación social, n. 72, pp. 1.536-1.553.

https://doi.org/10.4185/RLCS-2017-1233

Bremer, Laura (2013). Enhancing youth employability: What? Why? and How? Guide to core work skills. Génova: International Labour Office. Skills and Employability Department.

http://www.ilo.org/wcmsp5/groups/public/---ed_emp/--ifp_skills/documents/publication/wcms_213452.pdf

APM (2017). Informe anual de la profesión periodística. Madrid: Asociación de la Prensa de Madrid.

https://goo.gl/xt7QjV

Carey, Benedict (2018). "'Fake News': Wide reach but little impact, study suggests". The New York Times, January $2^{\text {nd }}$. https://www.nytimes.com/2018/01/02/health/fake-newsconservative-liberal.html?smid=tw-nytimes\&smtyp $=$ cur

Casero-Ripollés, Andreu; Cullell-March, Cristina (2013). "Periodismo emprendedor. Estrategias para incentivar el autoempleo periodístico como modelo de negocio". Estudios sobre el mensaje periodístico, v. 19, pp. 681-690.

https://doi.org/10.5209/rev_ESMP.2013.v19.42151

Casero-Ripollés, Andreu; Ortells-Badenes, Sara; Doménech-Fabregat, Hugo (2013). "Las competencias profesionales en periodismo: una evaluación comparativa". Historia y comunicación social, v. 18, pp. 53-64.

http://revistas.ucm.es/index.php/HICS/article/view/44311

Ciampaglia, Giovanni-Luca; Shiralkar, Prashant; Rocha, Luis M.; Bollen, Johan; Menczer, Filippo; Flammini, Alesandro (2015). "Computational fact checking from knowledge networks". PLoS one, v. 10, n. 6, e0128193.

https://doi.org/10.1371/journal.pone.0128193

Comisión Europea (2018). "Experts appointed to the high-level group on fake news and online disinformation".
Commission and its priorities, January, $12^{\text {th }}$.

https://ec.europa.eu/digital-single-market/en/news/ experts-appointed-high-level-group-fake-news-and-onlinedisinformation

Crucianelli, Sandra (2012). "Introducción al periodismo de datos". Ijnet: Red de periodistas internacionales. https://goo.gl/7Ynv4X

Eldiario.es (2016). “Ignacio Escolar: 'La prensa sufre una crisis de credibilidad como la de las instituciones'”. Eldiario.es, 14 de septiembre.

http://www.eldiario.es/c/m/Ignacio-Escolar-prensacredibilidad-instituciones_0_558894329.html

Elías-Pérez, Carlos (2015). Big data y periodismo en la sociedad red. España: Síntesis. ISBN: 9788490772034

Europa Press (2017). "Francisco Rosell, director de El Mundo: 'Las 'fake news' revalorizan, revitalizan el papel del periodismo". Europa Press, 4 octubre.

http://www.europapress.es/sociedad/noticia-franciscorosell-director-mundo-fake-news-revalorizan-revitalizanpapel-periodismo-20171004123810.html

Farias-Batlle, Pedro (dir.) (2009). Informe anual de la profesión periodística 2009. Madrid: Asociación de la Prensa de Madrid. Madrid. ISBN: 9788487641435

https://www.apmadrid.es/wp-content/uploads/2009/02/ Informe\%20APM\%202009.pdf

Fole, Xabier (2012). "Los hechos son sagrados. El fact-checker y la importancia del periodismo". Fronterad, 26 septiembre.

http://www.fronterad.com/?q=hechos-son-sagrados-factchecker-y-importancia-periodismo

Fundéu (2013). "Verificación, mejor que fact checking". Fundación para el Español Urgente, 21 junio.

https://www.fundeu.es/recomendacion/verificacion-mejorque-fact-checking

García-Ureta, Irene; Toral-Madariaga, Gotzon; Murelaga-Ibarra, Jon (2012). "Propuesta docente para la formación de comunicadores: desarrollo de competencias psicológicas y conexión con los retos profesionales". Estudios sobre el mensaje periodístico, v. 18, pp. 413-423.

https://doi.org/10.5209/rev_ESMP.2012.v18.40996

García-Valcárcel-Muñoz-Repiso, Ana; Martín-del-Pozo, Marta (2016). "Análisis de las competencias digitales de los graduados en titulaciones de maestro". Revista latinoamericana de tecnología educativa, v. 15, n. 2, pp. 155-168. https://doi.org/10.17398/1695-288X.15.2.155

Gómez-Mompart, Josep-Lluís; Gutiérrez-Lozano, JuanFrancisco; Palau-Sampio, Dolors (2015). "Los periodistas españoles y la pérdida de la calidad de la información: el juicio profesional". Comunicar, v. 23, n. 45, pp. 143-150. https://doi.org/10.3916/C45-2015-15

González-Molina, Sonia; Ortells-Badenes, Sara (2012). “La polivalencia periodística de los profesionales en las redes sociales". Estudios sobre el mensaje periodístico, v. 19, pp. 455-463.

https://doi.org/10.5209/rev_ESMP.2012.v18.41000 
Graves, Lucas (2016). Deciding what's true. The tise of political fact-checking in American journalism. Columbia University Press. ISBN: 9780231175074

Greenberg, David (2017). "Deciding what's true: The rise of political fact-checking in American journalism". Journal of communication, v. 67, n. 6.

https://doi.org/10.1111/jcom.12329

Haigh, Maria; Haigh, Thomas; Kozak, Nadine I. (2017). "Stopping fake news. The work practices of peer-to-peer counter propaganda". Journalism studies, Abril 25 $5^{\text {th }}$, pp. 1-26 https://doi.org/10.1080/1461670X.2017.1316681

Hazard-Owen, Laura (2018). “Here's a first attempt to quantify the extent of Europe's fake news problema". Nieman journalism lab, Jan. $31^{\text {st }}$.

http://www.niemanlab.org/2018/01/heres-a-first-attemptto-quantify-the-extent-of-europes-fake-news-problem/

Humanes-Humanes, María-Luisa; Roses-Campos, Sergio (2014). "Valoración de los estudiantes sobre la enseñanza del periodismo en España". Comunicar, v. 21, n. 42, pp. 181-188. https://doi.org/10.3916/C42-2014-18

Jankowski, Nicholas W. (2018). "Researching fake news: A selective examination of empirical studies". Journal of the European institute for communication and culture, v. 25, n. 1-2, pp. 248-255.

https://doi.org/10.1080/13183222.2018.1418964

Khaldarova, Irina; Pantti, Mervi (2016). "Fake news. The narrative battle over the Ukrainian conflict". Journalism practice, v. 10, n. 7, pp. 891-901.

https://doi.org/10.1080/17512786.2016.1163237

Kleis-Nielsen, Rasmus; Graves, Lucas (2017). News you don't believe: Audience perspectives on fake news. Reuters Institute for the Study of Journalism, with the support of Google and the Digital News Initiative, October.

https://goo.gl/8smGXQ

Kovach, Bill; Rosenstiel, Tom (2001). The elements of journalism: What news people should know and the public should expect. New York: Crown Publishers. ISBN: 9780 609504314

La sexta (2017). "Ana Pastor en la cumbre del periodismo fact-checking: 'Hace mucha falta que verifiquemos para que la gente cuando comparta la noticia pueda saber si es cierta o es falsa'". El objetivo, 28 octubre.

https://goo.gl/uCGUAn

Llaneras, Kiko; Pérez-Colomé, Jordi (2017). “España también tiene noticias falsas". El país, 28 enero.

https://politica.elpais.com/politica/2017/01/27/ actualidad/1485523499_326784.html

López-García, Xosé (2012). “La formación de los periodistas para los entornos digitales actuales". Revista de comunicación, n. 11, pp. 178195.

https://dialnet.unirioja.es/servlet/articulo?codigo $=4508642$

López-García, Xosé; Rodríguez-Vázquez, Ana-lsabel; Álvarez-Gromaz, Lucía (2016). "El fact checking como reclamo y como servicio en los cibermedios. Análisis de las experiencias de 'The Washington Post' y 'The Guardian'”.
Telos, n. 13.

https://goo.gl/KtMqgL

López-García, Xosé; Rodríguez-Vázquez, Ana-Isabel; Pereira-Fariña, Xosé (2017). "Competencias tecnológicas y nuevos perfiles profesionales: desafíos del periodismo actual". Comunicar, v. 25, n. 53.

https://doi.org/10.3916/C53-2017-08

López-García, Xosé; Toural-Bran, Carlos; Rodríguez-Vázquez, Ana-Isabel (2016). "Software, estadística y gestión de bases de datos en el perfil del periodista de datos". El profesional de la información, v. 25, n. 2, pp. 286-294.

https://doi.org/10.3145/epi.2016.mar.16

Manfredi-Sánchez, Juan-Luis (coord.) (2015). Innovación y periodismo: emprender en la universidad. Cuadernos artesanos de comunicación, n. 76. La Laguna, Tenerife: Sociedad Latina de Comunicación Social. ISBN: 9788415698937

Manfredi-Sánchez, Juan-Luis; Rojas-Torrijos, José-Luis; Herranz-de-la-Casa, José-María (2015). "Periodismo emprendedor: el periodismo deportivo en España". Revista latina de comunicación social, n. 70, pp. 69-90.

https://doi.org/10.4185/RLCS-2015-1035

Margolin, Drew B.; Hannak, Aniko; Weber, Ingmar (2017). "Political fact-checking on Twitter: When do corrections have an effect?" Political communication, Sept. $5^{\text {th }}$ https://doi.org/10.1080/10584609.2017.1334018

Martín-del-Peso, Mónica; Rabadán-Gómez, Ana-Belén; Hernández-March, Julio (2013). "Desajustes entre formación y empleo en el ámbito de las enseñanzas técnicas universitarias: la visión de los empleadores de la Comunidad de Madrid". Revista de educación, n. 360, pp. 244-267. https://doi.org/10.4438/1988-592X-RE-2011-360-110

Massot, Josep (2017). 'Fake news': cuando la opinión vale más que los hechos. La vanguardia, 26 diciembre. https://goo.gl/KYcoMK

Meyer, Philip (1991). The new precision journalism. Bloomington: Indiana University Press. ISBN: 9780253206640

Nelson, Jacob L.; Harsh, Taneja (2018). "The small, disloyal fake news audience: The role of audience availability in fake news consumption". Communication research, Febr. $18^{\text {th }}$. https://doi.org/10.1177/1461444818758715

Palomo, Bella (2013). "Claves de la implantación y la expansión del perfil social del periodista". Comunicación y medios, n. 28, pp. 113129.

https://comunicacionymedios.uchile.cl/index.php/RCM/ article/view/27403

Perlado-Lamo-de-Espinosa, Marta; Rubio-Romero, Juana (2015). "Competencias, habilidades y formación del creativo publicitario en la era digital". Creatividad y sociedad, n. 23, pp. 6-34.

https://goo.gl/utXHXV

Redondo, Myriam (2017). "Buenas noticias sobre fact-checking y verificación". Globograma, periodismo internacional, relaciones internacionales, 15 enero.

http://www.globograma.es/buenas-noticias-sobre-factchecking-y-verificacion 
Renó, Luciana; Flores, Jesús (2014): “Periodismo de datos en el mundo transmedia". En: Campalans, Carolina; Renó, Denis; Gosciola, Vicente. Narrativas transmedia: entre teorías y prácticas. Barcelona: Oberta Press. ISBN: 9788490642382

Sánchez-González, Hada; Méndez-Muros, Sandra (2013). "¿Perfiles profesionales 2.0? Una aproximación a la correlación entre la demanda laboral y la formación universitaria". Estudios sobre el mensaje periodístico, v. 19, pp. 981-993. https://doi.org/10.5209/rev_ESMP.2013.v19.42183

Sanhermelando, Juan (2018). "Bruselas busca soluciones para frenar la propagación de 'fake news'”. El español, 15 enero.

https://www.elespanol.com/economia/medios/20180115/ bruselas-busca-soluciones-frenar-propagacion-fakenews/277472646_0.html

Tandoc, Edson; Lim, Zheng-Wei; Ling, Richard (2017) "Defining "fake news": A typology of scholarly definitions". Digital journalism, v. 6, n. 2, pp. 137-153. https://doi.org/10.1080/21670811.2017.1360143

Torres-Coronas, Teresa; Vidal-Blasco, María-Arántzazu (2015). "Percepción de estudiantes y empleadores sobre el desarrollo de competencias digitales en la educación superior". Revista de educación, n. 367, pp. 63-90.

https://doi.org/10.4438/1988-592X-RE-2015-367-283

Uscinski, Joseph E.; Butler, Ryden W. (2013). "The epistemology of fact checking". Critical review. A journal of politics and society, v. 25, n. 2, pp. 162-180.

https://doi.org/10.1080/08913811.2013.843872

Wilkinson, John-William (2017). “El más que rancio abolengo de las 'fake news'". La vanguardia, 9 de diciembre. https://goo.gl/cC7Bfe

Wintersieck, Amanda L. (2017). "Debating the truth. The impact of fact-checking during electoral debates". American politics research, v. 45, n. 2, pp. 304-331.

https://doi.org/10.1177/1532673X16686555

\section{Dialnet visbitidad par lo producción científica}

en español

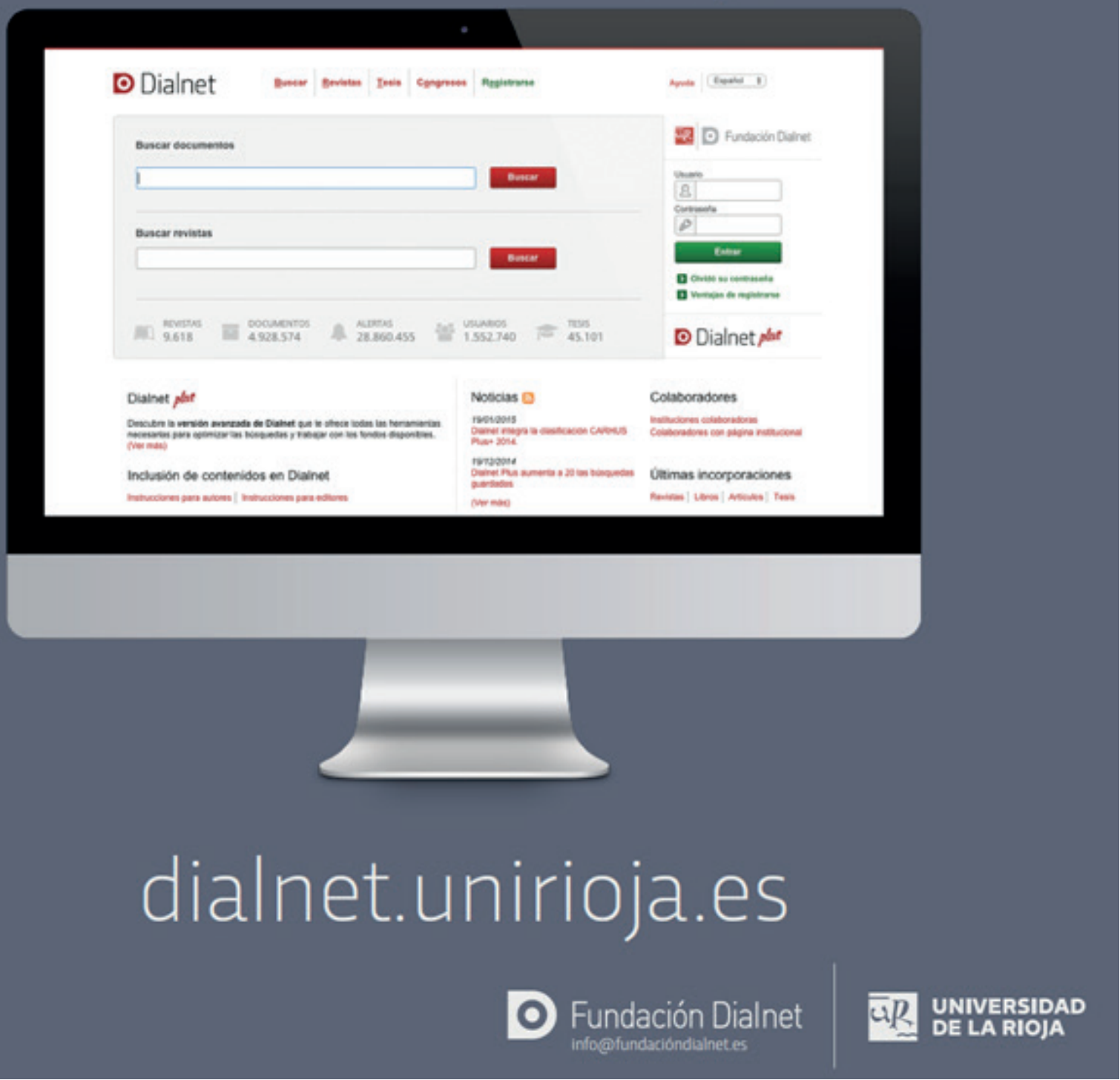

